Tracy Camille P. Chan, MD

Ma. Clarissa S. Fortuna, MD

Patrick S. Enriquez MD

Department of Otorhinolaryngology

Head and Neck Surgery

The Medical City
Correspondence: Dr. Ma. Clarissa S. Fortuna Department of Otorhinolaryngology

Head and Neck Surgery

The Medical City

Ortigas Avenue, Pasig City 1600

Philippines

Phone: (632) 6356789 local 6250

Fax: (632) 6873349

Email: ent@medicalcity.com.ph

The authors declare that this represents original material, that the manuscript has been read and approved by all the authors, that the requirements for authorship have been met by each author, and that each author believes that the manuscript represents honest work.

Disclosures: The authors signed disclosures that there are no financial or other (including personal) relationships, intellectual passion, political or religious beliefs, and institutional affiliations that might lead to a conflict of interest.

Presented at the Philippine Society of Otolaryngology Head and Neck Surgery Descriptive Research Contest (3rd Place), September 24, 2015. Natrapharm, The Patriot Bldg., Paranaque City, Philippines.

\section{(1) $\Theta \Theta$}

\title{
Demographic Profile and Risk Factors of Patients with Benign Vocal Fold Lesions Diagnosed through Laryngeal Videoendoscopy and Stroboscopy
}

\section{ABSTRACT}

Objective: To determine the prevalence of benign vocal cord lesions among Filipino patients in a tertiary institution and identify the demographic characteristics and possible risk factors found among these patients.

Methods:

\section{Design: $\quad$ Retrospective Case Series}

Setting: $\quad$ Private Tertiary Hospital

Participants: Records of 2,375 patients who underwent laryngeal video endoscopy and stroboscopy from 2012-2014 were reviewed.

Results: There were 632 records of patients with benign vocal fold lesions, of which nodules were most common $(211,33.38 \%)$ followed by Reinke's edema $(165,26.10 \%)$, cysts $(122,19.30 \%)$ and polyps $(74,11.70 \%)$ with hoarseness as the most common symptom $(542,85.76 \%)$. More than half $(336,53.16 \%)$ were aged $21-40$ years and almost two-thirds $(469,74.21 \%)$ were female. The most common associated factors were caffeine intake $(445,70.41 \%)$ and inadequate water intake $(370,58.54 \%)$, followed by alcohol $(253,40.03 \%)$. Smoking was only present in $146(23.19 \%)$.

Conclusions: Baseline evidence on the prevalence of benign vocal fold lesions in this institution as well as baseline data on the common characteristics and associated factors seen in the sample population may assist us in current practices and guide future studies directed toward treatment and prevention.

Keywords: Vocal Cord; Stroboscopy; Vocal Cord Nodules; Benign Vocal Cord; Stroboscopy/Benign; Stroboscopy/Nodules

Current advances in technology have led to the development of tools for easier visualization and diagnosis of the larynx. Particularly useful is laryngeal videoendoscopy and stroboscopy (LVES). ${ }^{1,2}$ Over $50 \%$ of patients presenting with voice complaints have have benign vocal cord lesions, ${ }^{1,3-5}$ among which nodules are most prevalent, ${ }^{2,5,7}$ followed by vocal cord polyps, Reinke's edema, cysts and papillomas., ${ }^{8} 9$ Hoarseness is the most frequent presenting symptom although other symptoms such as cough, foreign body sensation, heartburn, throat-clearing, pain, breathlessness and vocal breaks are also noted. ${ }^{2,5,7}$ Benign vocal cord lesions are more often diagnosed in males than females and are more often found in the 20-60 year old age group. ${ }^{5,8}$ Commonalities include talkative personalities and voice-requiring occupations related to voice 


\section{ORIGINAL ARTICLES}

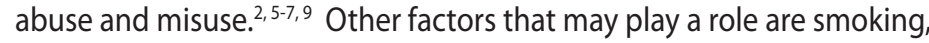
alcohol intake, reflux, allergies, chronic cough and infection, 5 , with smoking contributing heavily to the appearance of lesions. ${ }^{8}$ However, other data has shown no significant relationship between smoking and the presence of benign vocal cord lesions. ${ }^{6}$

Moreover, data about the prevalence of benign vocal cord lesions as well as the patient profiles and postulated risk factors associated with them are available in foreign but not local literature and it is important to establish if such data is applicable and comparable to local data.

This study seeks to determine the prevalence of benign vocal cord lesions among Filipino patients in a tertiary institution and identify the demographic characteristics and possible risk factors found among these patients.

\section{METHODS}

With institutional review board protocol approval, a retrospective review of records of all patients who underwent laryngeal videoendoscopy and stroboscopy (LVES) at the Voice and Swallowing Laboratory of our tertiary hospital from January 1, 2012 to December 31,2014 , and were subsequently diagnosed to have benign vocal cord lesions (nodules, cysts, polyps, granulomas, Reinke's edema, papillomas) were considered for inclusion. Excluded were records of caucasian patients, lesions that were diagnosed as neither benign nor malignant (i.e. atrophy, varices, web), overtly malignant lesions and lesions of infectious etiology.

Primary sources of data included patient questionnaires and printed-out LVES results. Prior to undergoing LVES, patients were routinely interviewed using the questionnaire. Data obtained from this questionnaire included the presenting symptom, age, sex, occupation, prior treatment, concomitant medical conditions and risk factors. All LVES had been conducted using a Kay RLS 9100 Rhino-Laryngeal Stroboscope and Digital Videostroboscopy System (Kay Elemetrics, NJ, USA). Printouts included pertinent images and the subsequent diagnosis.

Data on voice usage was excluded from the study as this was a subjective factor and could not be quantified. The Reflux Symptom Index (RSI) was also excluded as this was considered more relevant for reflux as a separate disease entity.

Patient anonymity was protected in the collection of data. Confidential patient data was only known to the authors who were also the physicians who conducted LVES examinations.

Data was compiled and analyzed with descriptive statistics using Microsoft Excel for Mac 2011 Version 14.6.8 (Microsoft Corporation, Redmond, WA, USA).
A total of 2,375 patient records were reviewed for inclusion in this study. Of these, 632 had a diagnosis of benign vocal cord lesions and were included. Excluded were 1,743 records; 265 with laryngopharyngeal reflux (LPR), 688 with other laryngeal pathologies (i.e. infectious, cancer), 442 with non-glottic/non-laryngeal pathologies, 84 with normal laryngeal findings, 201 without a written diagnosis and 63 with insufficient data.

Of the 632 patients included, the majority $(178,28.16 \%)$ belonged to the 21-30 year old age bracket closely followed by the 31-40 year-old $(158,25.00 \%)$, and $41-50$ year-old $(131,20.73 \%)$ age groups. There were thrice more females $(469,74.21 \%)$ than males $(160,25.32 \%)$.

The most common occupations were customer service representative $(200,31.64 \%)$ and office employee $(64,10.12 \%)$. Other occupations included teachers $(49,7.75 \%)$, housewives $(29,4.58 \%)$ and businessmen $(27,4.27 \%)$.

Of the benign vocal cord lesions, nodules were most common (211, $33.38 \%)$ followed by Reinke's edema (165, 26.10\%), cysts (122, 19.30\%), and polyps $(74,11.70 \%)$. Least common were laryngeal papilloma (7, $1.10 \%)$, sulcus $(26,4.11 \%)$ and granuloma $(27,4.27 \%)$. A total of 246 (38.92\%) were found to have concomitant LPR.

The majority presented with hoarseness $(542,85.76 \%)$ followed less commonly by pain $(22,3.48 \%)$, globus sensation $(13,2.06 \%)$ and throat discomfort $(13,2.06 \%)$. Aside from their primary diagnosis, 353 (55.85\%) had a past history of other illnesses (i.e. GERD, rhinitis, asthma), and 257 (40.66\%) had received some form of treatment (i.e. antibiotics, antihistamines, PPI) prior to the evaluation.

Among the risk factors evaluated, the most common were caffeine intake $(445,70.41 \%)$ and inadequate water intake (370, 58.54\%) followed by alcohol (253, 40.03\%). Surprisingly, smoking was only present in 146 (23.19\%).

\section{DISCUSSION}

Consistent with previous studies, 5,8 benign lesions were predominantly found in the 20-60 year-old age group. However, unlike these studies ${ }^{5,8}$ benign lesions in our study were more predominant in females than in males.

Also consistent with previous studies, 2,5,7 vocal cord nodules were the most common of the benign vocal cord lesions. However, certain differences were noted in this study that may be attributed to differences in culture and possibly to the local surge in call center workers whose profession requires long hours of voice use. The second most common benign lesion in this study was Reinke's edema followed by cysts then polyps in contrast to studies elsewhere ${ }^{8,9}$ where polyps are second most common followed by Reinke's edema and cysts. 
It is well established that benign lesions are most frequently diagnosed in those with voice abuse related occupations. ${ }^{2,5,7,9}$ This was affirmed in our study where a large number were customer service representatives whose jobs are very much voice-dependent. Our finding of hoarseness as the most common presenting symptom is also consistent with the literature. ${ }^{2,5,7}$

On the other hand, contrary to previous reports, ${ }^{5,7,8}$ caffeine intake, rather than smoking, was the most common risk factor in our study.

This study was intended to benefit both clinicians and professionals in the academe who wish to acquire locally - based descriptive data about the prevalence and demographic profile of patients with benign vocal cord disorders.

The study is limited in that despite the availability of data with which may be inadequate and should not be considered a representative sample of the population. This may be resolved by extending the period of the study to more than just 5 years or by increasing the number of subjects by obtaining data from other institutions/facilities.

In conclusion, laryngeal videoendoscopy and stroboscopy plays a valuable role in the detection of vocal pathologies and has enabled us to gather baseline evidence on the prevalence of benign vocal fold lesions in our institution as well as baseline data on the common characteristics and associated factors seen in the sample population. Knowing these factors may assist us in current practices and guide future studies directed toward the treatment and prevention of these lesions.
REFERENCES

1. Samlan RA, Gartner-Schmidt J, Kunduk M. Visualization of the Larynx. In Flint PW, Haughey BH, Lund VJ, Niparko JK, Richardson MA, Robbins KT, et al (editors). Cummings Otolaryngology Head and Neck Surgery. 5th ed., Vol. 1. Philadelphia, PA: Elsevier- Mosby. 2010. pp.813-824.

2. Yogesh S, Daharwal A, Prasad R, Singh S, Shankari -Raipur V. Shankari -Raipur (Chhatisgarh). Videostroboscopy study of larynx in primary school teachers. National Journal of Otolaryngology and Head and Neck Surgery. 2014 Jan; 11(1), 32-33. [Retrieved 2015 Feb 13]. Available from: https://www.researchgate.net/publication/281889037_Videostroboscopy_study_of_larynx_ in_primary_school_teachers.

3. Bohlender J. Diagnostic and therapeutic pitfalls in benign vocal fold diseases. GMS Curr Top Otorhinolaryngol Head Neck Surg. 2013 Dec 13; 12: Doc01. [Retrieved 2015 Feb 14]. Available from http://www.egms.de/static/pdf/journals/cto/2013-12/cto000093.pdf. DOI: 10.3205/ cto000093; PMID: 24403969; PMCID: PMC3884536.

4. Dabirmoghadam $P$, Azimian S, Mokhtari Z. Stroboscopic findings in patients with benign laryngeal lesions: A brief report. Tehran Univ Med J. 2012 Nov; 70(8): 508-513. [Retrieved 2015 Feb 13]. Available from http://tumj.tums.ac.ir/browse.php?a_id=88\&sid=1\&slc_lang=en.

5. Wani AA, Rehman A, Hamid S, Akhter M, Baseena S. Benign mucosal fold lesion as a cause of hoarseness of voice-a clinical study. Otolaryngology. 2012:2(3). [Retrieved 2015Feb 13]. Available from: https://www.omicsonline.org/benign-mucosal-fold-lesion-as-a-cause-of-hoarseness-ofvoice-a-clinical-study-2161-119X.1000120.pdf. DOI:10.4172/2161-119X.1000120.

6. Byeon $\mathrm{H}$. Exploring potential risk factors for benign vocal fold mucosal disorders using weighted logistic regression. International Journal of Bio-Science and Bio-Technology. 2014; 6(4), 77-86. [Retrieved 2015 Feb 13]. Available from: http://www.sersc.org/journals/IJBSBT/vol6_no4/8.pdf. DOI: dx.doi.org/10.14257/ijbsbt.2014.6.4.08.

7. Smith S, Underbrink M. Benign vocal fold lesions. Grand Rounds Presentation, The University of Texas Medical Branch in Galveston, Department of Otolaryngology. 2013 Nov 26. [Retrieved 2015 Feb 13]. Available from http://www.utmb.edu/otoref/GRNDS/vocal-cord-benign-lesions2013-11/vocal-cord-2013-11.pdf.

8. Gupta N, Gurnani D, Patel N, Patel T, Sharma P, Jindal S, et al. Benign vocal cord lesions. National Journal of Otorhinolaryngology and Head \& Neck Surgery. 2013 Jan; 1(2): 19-20. [Retrieved 2015 Feb 13]. Available from: https://www.researchgate.net/publication/291911634_Benign vocal_cord_lesions.

9. Nunes RB, Behlau M, Nunes MB, Paulino JG. Clinical diagnosis and histological analysis of vocal nodules and polyps. Braz J Otorhinolaryngol. 2013 Aug; 79(4): 434-440. [Retrieved 2015 Feb 14]. Available from http://www.scielo.br/pdf/bjorl/v79n4/en_v79n4a07.pdf. DOI: 10.5935/18088694.2013007: PMID: 23929142

10.Perez Fernandez CA, Preciado Lopez J. Vocal fold nodules, risk factors in teachers. A case control study design. Acta Otorrinolaringol Esp. 2003 Apr; 54(4): 253-260. [Retrieved 2015 Feb 14]. Available from http://www.ncbi.nlm.nih.gov/pubmed/12825241. PMID: 12825241. 